\title{
De la percepción a los usos del tiempo: perspectiva temporal y procrastinación de adultos en España*
}

\section{From the perception to the uses of time: Time perspective and procrastination among adults in Spain}

\author{
Dra. Nuria CODINA. Profesora Titular. Universitat de Barcelona (ncodina@ub.edu). \\ Dr. Rafael VALENZUELA. Profesor Asociado. Universitat de Barcelona (rvalenzuela@ub.edu). \\ Dr. José Vicente PESTANA. Profesor Agregado. Universitat de Barcelona (jvpestana@ub.edu).
}

\section{Resumen:}

Las ciencias del comportamiento han investigado desde diferentes perspectivas la relación entre el dominio o control del tiempo y el desarrollo humano, perfilándose, en las últimas décadas, dos manifestaciones actitudinales altamente implicadas en dicho desarrollo: las orientaciones temporales y la procrastinación. Respecto a estos conceptos abunda la literatura; sin embargo, pocos trabajos aportan datos relativos a la relación entre ambas actitudes y aspectos de la vida cotidiana datos que pueden poner sobre aviso opciones para regular dichas actitudes-. En esta investigación se analizan la perspectiva temporal y la procrastinación en relación con la edad, las caracterís- ticas temporales del trabajo y las condiciones de cohabitación (con quién se vive). Participaron 720 adultos (390 hombres y 330 mujeres) con edades comprendidas entre 18 y 64 años $(M=40.44 ; D T$ = 9.80). Los instrumentos utilizados fueron un cuestionario ad hoc de datos sociodemográficos y dos escalas validadas para población española: el Inventario de Perspectiva Temporal de Zimbardo y el instrumento de procrastinación que integra la General Procrastination Scale, el Decisional Procrastination Questionnaire y el Adult Inventory of Procrastination. Los resultados muestran una perspectiva general no equilibrada en el conjunto de la muestra, observándose relaciones significativas según la edad, la situación de cohabitación

\footnotetext{
* Este trabajo se vincula al Proyecto de Investigación «Gestión del tiempo y autonomía en jóvenes: fomentando competencias no técnicas» (RTI2018-094405-B-I00), cofinanciado en el marco del Programa estatal de I+D+i con cargo a una ayuda del Ministerio de Ciencia, Innovación y Universidades y por el Fondo Europeo de Desarrollo Regional. Investigadores principales: N. Codina y J. V. Pestana.
}

Fecha de recepción de la versión definitiva de este artículo: 08-06-2020.

Cómo citar este artículo: Codina, N., Valenzuela, R., Pestana, J. V. (2020). De la percepción a los usos del tiempo: perspectiva temporal y procrastinación de adultos en España $\mid$ From the perception to the uses of time: Time perspective and procrastination among adults in Spain. Revista Española de Pedagogía, 78 (277), 435-456. doi: https://doi. org/10.22550/REP78-3-2020-04

https://revistadepedagogia.org/

ISSN: 0034-9461 (Impreso), 2174-0909 (Online) 
(p. e., más pasado negativo $-d=.33-\mathrm{y}$ presente hedonista $-d=.30-$ en quienes viven con sus padres) y las características temporales del trabajo (p. e., más presente fatalista en quienes tienen unos horarios rígidos $-d=.53$ ). La procrastinación está significativamente más presente en los jóvenes entre 18-29 años (en los factores de conductas dilatorias $-d=.63-\mathrm{y}$ falta de puntualidad $-d=.69$ ); asimismo, varios de sus factores están asociados a vivir solo 0 al hecho de alargar la jornada laboral. Estos hallazgos apuntan unos condicionantes relacionados con manifestaciones actitudinales hacia el tiempo, resultados que apuntan variables específicas de la cotidianidad sobre las cuales se puede intervenir con el objetivo de facilitar el desarrollo de personas con potencial para dominar o controlar el tiempo.

Descriptores: procrastinación, perspectiva temporal, cohabitación, edad, trabajo.

\section{Abstract:}

The behavioural sciences have investigated the relationship between time domain or control and human development from a variety of perspectives, in recent decades, outlining two attitudinal manifestations that are deeply involved in such development: time orientations and procrastination. There is abundant literature regarding these concepts, but few works provide data about the relationship between these attitudes and aspects of everyday life, data that might identify options to regulate such attitudes. This paper anal- yses time perspective and procrastination with regards to age, temporal characteristics of work, and living arrangements. 720 adults ( 390 men and 330 women) aged between 18 and 64 years $(M=$ $40.44 ; S D=9.80$ ) participated. The instruments used were an ad hoc questionnaire on sociodemographic data, and two scales validated for the Spanish population: the Zimbardo Time Perspective Inventory and a procrastination instrument that combines the General Procrastination Scale, the Decisional Procrastination Questionnaire, and the Adult Inventory of Procrastination. The results show an unbalanced general perspective in the sample as a whole, with significant relationships by age, living arrangements (e.g., more negative past $-d=.33$ - and hedonistic present $-d=.30-$ among respondents who live with their parents) and temporal characteristics of work (e.g., more fatalistic present in respondents with rigid schedules $-d=.53$ ). Procrastination is more present in young people aged between 18 and 29 (in the dilatory behaviour $-d=.63$ - and lack of punctuality $-d=.69-$ factors). Also, several of these factors are associated with living alone or with extending the working day. These findings highlight determining factors relating to attitudes to time, results that highlight specific variables from daily life which can be the subject of interventions to facilitate the development of people with the potential to dominate or control time.

Keywords: procrastination, time perspective, living arrangements, age, work.

\section{Introducción}

Desde la perspectiva del desarrollo humano, entendemos que dominar o contro- lar el tiempo es sobreponerlo a la propia voluntad. Este cometido, en las ciencias del comportamiento, se estudia a través de pro- 
cesos que van desde las estrategias de gestión del tiempo hasta la autorregulación del tiempo — pasando por las actitudes hacia el tiempo o el problema de la procrastinación.

Las investigaciones sobre estos temas aportan gran cantidad de conocimiento; sin embargo, pocos trabajos aportan información específica respecto a la relación entre actitudes hacia el tiempo y variables situacionales (ocupación principal, horarios 0 condiciones de convivencia). En consecuencia, las valoraciones de los dominios del tiempo y los diseños de intervención se hacen desde estándares que no contemplan pormenores de la realidad que se estudia -a pesar de que sí inciden en el dominio del tiempo que presentan las personas.

Frente a estas formas de estudiar el tiempo, el presente trabajo se centra en las orientaciones temporales y las tendencias procrastinadoras de personas trabajadoras en relación con la edad, las características temporales del trabajo (horas trabajadas y criterios de flexibilización temporal) y los tipos de cohabitación; todo ello, con el objetivo de mostrar cómo inciden estas variables en el dominio o control del tiempo - y que deben ser consideradas en los estudios e intervenciones sobre este dominio o control.

\subsection{La perspectiva temporal}

Las orientaciones temporales se comportan como actitudes hacia el tiempo, las cuales - a menudo de manera no consciente- constituyen una forma de dominar 0 controlar el tiempo, condicionan el comportamiento (Zimbardo y Boyd, 1999) y juegan un papel decisivo en el bienestar de las personas (Boniwell, Osin, Linley e Ivan- chenko, 2010; Drake, Duncan, Sutherland, Abernethy y Henry, 2008; Simons, Peeters, Janssens, Lataster y Jacobs, 2018). Estas actitudes se han venido estudiando a través de las denominaciones de perspectiva temporal y orientación temporal.

La perspectiva temporal hace referencia fundamentalmente a la distancia cognitiva a la que se sitúan las experiencias vividas y las metas (Nuttin, 1985) y la orientación temporal, a la tendencia diferencial del sujeto a estar centrado en el pasado, presente o futuro (Lewin, 1948). No obstante, en los últimos años y siguiendo uno de los modelos teóricos más influyentes —el de Zimbardo y Boyd (1999) — muchos autores (incluidos los de este trabajo) utilizan ambas expresiones indistintamente para referirse al segundo concepto. Según dicho modelo, la orientación temporal es un proceso situado en el origen del comportamiento individual y social, que codifica, organiza y recuerda experiencias vividas y construye nuevas metas, expectativas y escenarios futuros.

De acuerdo con la teoría de Zimbardo (Zimbardo y Boyd, 2009; Zimbardo, Keough y Boyd, 1997), la perspectiva temporal de las personas está integrada por las siguientes cinco dimensiones y sus correspondientes actitudes: 1) el pasado negativo, que refleja una actitud pesimista, negativa 0 aversiva hacia el pasado; 2) el pasado positivo, que se expresa con una nostalgia y construcción positiva del pasado; 3) el presente hedonista, que lleva a vivir al día, buscar la gratificación inmediata y el placer; 4) el presente fatalista, se asocia a una cierta desesperación respecto al futuro e imposibilidad de esperar un futuro placentero a partir del comporta- 
miento presente; y 5) el futuro, relacionado con el logro de objetivos futuros, la gratificación retardada y la evitación de la pérdida de tiempo (Zimbardo y Boyd, 2009).

Estas orientaciones temporales tienen un protagonismo desigual en la dinámica de la perspectiva temporal de las personas, dando lugar a actitudes temporales que pueden inhibir o favorecer el desarrollo de la persona. Específicamente, el predominio de una dimensión por encima de las otras propicia actitudes y estilos disposicionales particulares.

Así, si la dimensión predominante es el pasado negativo o positivo, se tiende a actuar en respuesta a situaciones recurrentes que reflejan experiencias pasadas y a presentar manifestaciones de ansiedad y afecto negativo (D'Alessio, Guarino, De Pascalis, y Zimbardo, 2003, Drake et al., 2008); si predomina el presente fatalista, se es propenso a creer que el futuro está predestinado y que hay que vivir con resignación (Zimbardo y Boyd, 2009); si predomina el presente hedonista, se inclinan a pensar que lo importante es vivir el presente, sin importar las consecuencias futuras (Zimbardo y Boyd, 2009), pero si el predominio del presente es muy intenso, entonces lleva a aumentar la procrastinación, impulsividad y agresión (Ferrari y Díaz-Morales, 2007); si predominan los presentes fatalista y hedonista, la tendencia es sentirse bien y seguro en diferentes entornos; y, finalmente, si predomina el futuro, la persona procura orientar el comportamiento de acuerdo con los objetivos propuestos y sus beneficios, así como planificar su tiempo y sus actividades (Ferrari y Díaz-Morales, 2007; Shell y Husman, 2001, Zimbardo y Boyd, 2009).
Más allá de estos estilos disposicionales - por el dominio específico de una dimensión- se ha visto que cuando se presentan niveles más bajos en las orientaciones disfuncionales (pasado negativo y presente fatalista), más altos en las funcionales (pasado positivo y futuro) y niveles moderados en la de presente hedonista, se da una perspectiva temporal equilibrada u óptima, esto es, las personas tienden a experimentar mayor bienestar y mejor capacidad adaptativa (Boniwell y Zimbardo, 2004; Sircova et al., 2014; Boniwell et al., 2010; Drake et al., 2008; Webster, 2011; Wiberg, Sircova, Wiberg y Carelli, 2012). La importancia de mantener este equilibrio ha dado lugar a que a esta situación se la denomine competencia temporal (Zaleski, 1994): una competencia que hace que la persona afronte - con un menor coste psicológico y mayor éxito - tanto situaciones adversas como situaciones exitosas de la vida. Por lo tanto, en la dinámica de las cinco perspectivas temporales parece que una combinación deseable es la balanceada $\longrightarrow$ en su defecto, una dinámica en la que destaque la dimensión de futuro.

Aunque la investigación sobre las orientaciones temporales es abundante, los estudiosos reconocen que los resultados, a menudo, son inconsistentes y contradictorios — debido a la diversidad de variables y planteamientos de estudio (Kooij, Kanfer, Betts y Rudolph, 2018)_; y, en esta línea, argumentan que se necesita más investigación específica y mayor reflexión respecto a las variables sociodemográficas y los valores socioculturales que influyen en la perspectiva temporal de las personas $(\mathrm{Co}$ dina, Pestana y Ponce de León, 2018; Levasseur, Shipp, Fried, Rousseau y Zimbar- 
do, 2020; Soylu y Ozekes, 2019; Stolarski, Wiberg y Osin, 2015). Respecto a estos retos, algunos apuntan, específicamente, la necesidad de profundizar en el estudio del fenómeno en relación con las variables que nos proponemos trabajar: edad (Codina y Pestana, 2016; Laureiro-Martínez, Trujillo y Unda, 2017; Matthews y Stolarski, 2015), características temporales del trabajo (Bluedorn, 2002) y cohabitación (que, hasta donde sabemos, no cuenta con estudios de una cierta relevancia).

\subsection{La procrastinación}

Por lo que se refiere a la procrastinación, esta es un problema de dominio o control del tiempo consistente en el hábito de demorar -el inicio, la finalización o ambas - una tarea 0 actividad que se tenía la intención de realizar (Lay, 1986). Para más detalle, la procrastinación se manifiesta a través de cuatro tipos de comportamientos (Díaz-Morales, Ferrari, Díaz y Argumedo, 2006): 1) dilación, que consiste en el aplazamiento de la ejecución de la conducta que se tiene la intención de realizar; 2) indecisión, que se concreta en la postergación de decisiones dentro de un marco de tiempo específico; 3) falta de puntualidad, que se manifiesta con la incapacidad de cumplir satisfactoriamente con compromisos temporales; y 4) falta de planificación, que se revela con la falta de autodisciplina enfocada hacia una tarea específica. El hábito procrastinador, en cualesquiera de las dimensiones apuntadas, se expresa - entre otros ámbitos- en la escuela, la universidad, el trabajo, la salud, las rutinas diarias, la familia, la vida social o los trámites administrativos (Klingsieck, 2013), e incluso en el ocio (Pestana, Codina y Valenzuela, 2020).
Si bien la procrastinación es conocida y practicada en situaciones concretas por la mayoría de personas, cuando se convierte en un hábito o se generaliza, acarrea serios problemas personales, interpersonales y sociales (Goroshit, 2018). Por sus incidencias sobre la salud y el desarrollo - y por ser una práctica que sigue más del $20 \%$ de la población adulta (Harriott y Ferrari, 1996; Díaz-Morales y Ferrari, 2015) — la procrastinación ha estimulado una gran cantidad de los estudios sobre su naturaleza. Así, se ha investigado desde la incidencia de variables de personalidad (Kim, Fernández y Terrier, 2017; Steel, 2007), hasta variables psicosociales, como los estilos docentes, pasando por variables situacionales temporales, como la presión, la escasez o las regularidades (Codina, Castillo, Pestana y Balaguer, 2020; Codina, Valenzuela, Pestana y González-Conde, 2018; Valenzuela, Codina, Castillo y Pestana, 2020; Valenzuela, Codina y Pestana, 2020). Respecto a la cuestión de las variables situacionales, sin embargo, la investigación apenas ha considerado la incidencia de variables estructuradoras de la vida cotidiana, como son las características temporales del trabajo o la cohabitación; variables que pueden comportar ciertas regularidades que incidirían negativamente en la procrastinación.

\subsection{El presente estudio}

De acuerdo con estos antecedentes, en este trabajo se propone mostrar cómo las perspectivas temporales y la procrastinación se relacionan con variables personales y situacionales como la edad, la cohabitación y las características temporales del trabajo; objetivo con el que pretendemos enriquecer el cuerpo de conocimientos acerca de estos dos procesos y, con ello, reclamar la inclu- 
sión de estas variables en la investigación y orientar las estrategias de intervención.

\section{Método}

El presente estudio corresponde a una investigación no experimental del tipo asociativa (correlacional) y transversal (Ato, López y Benavente, 2013). En este sentido, la relación observada entre las variables se orienta hacia la comparación de grupos, esto es, explicitar en qué sectores de la población — de acuerdo con las variables estudiadas- se observan diferencias de acuerdo con las perspectivas temporales y los factores de la procrastinación.

\subsection{Participantes}

Datos sociodemográficos. Se obtuvieron mediante el cuestionario que registró: el sexo, la edad, las condiciones de cohabitación de los participantes (vivir solo, 0 bien con hijos / padres / personas a cargo) y la organización de la temporalidad en el entorno laboral (tiempo destinado a desplazarse al lugar de trabajo, horas trabajadas, flexibilidad al entrar y salir del trabajo y la prolongación de la jornada laboral).

Perspectiva temporal. Se analizó a través del Inventario de Perspectiva Temporal de Zimbardo (ZPTI: Zimbardo y Boyd, 1999), adaptado para la población española por Díaz-Morales (2006). Este instrumento está integrado por 56 ítems relativos a cinco dimensiones (para cada una de las cuales se pone un ejemplo del propio inventario): dos relativas al presente (hedonista: «Cuando escucho mi música favorita, pierdo la noción del tiempo»; y fatalista: «La vida de hoy es demasiado complicada, preferiría la vida más sencilla de antes»); dos, al pasado (positivo: «Me gustan los rituales y tradiciones familiares que se repiten regularmente»; y negativo: «Las experiencias dolorosas del pasado permanecen en mi memoria»); y una al futuro («Preparar el trabajo para el día siguiente y cumplir con los plazos, se antepone a la diversión de hoy en la noche» sin distinción de extremos positivo y negativo-). El formato de respuesta es del tipo Likert con cinco opciones de respuesta (que van desde el 1 - «no me describe en absoluto»— hasta el 5 - «muy característico»—). El alfa de Cronbach observado fue aceptable $(\alpha=.87)$ e incluso ligeramente superior a los valores obtenidos por Díaz-Morales (2006), que estuvieron entre .74 y 82 .

Procrastinación. Se midió a través de un instrumento de 40 ítems validado para la 
población española por Díaz-Morales et al. (2006). Esta prueba está integrada por tres cuestionarios (en cada caso, se especifican los valores del alfa de Cronbach obtenidos por Díaz-Morales et al., 2006): la General Procrastination Scale (GP: Lay, 1986; $\alpha_{\mathrm{GP}}=$ .84), el Decisional Procrastination Questionnaire (DP: Mann, 1982; $\alpha_{\mathrm{DP}}=.83$ ) y el Adult Inventory of Procrastination (AIP: McCown y Johnson, 1989; $\alpha_{\text {AIP }}=.81$ ). La integración de estas pruebas suman un total de 40 ítems, los cuales corresponden a cuatro factores (en cada caso, con un ejemplo de cada factor): conductas dilatorias («Tardo varios días en realizar trabajos, incluso los que solo requieren sentarse y hacerlos»), indecisión ( Retraso tanto mis decisiones que cuando me decido, ya es demasiado tarde»), falta de puntualidad («Mis amigos y mi familia piensan que siempre espero hasta el último minuto»), y falta de planificación («Preparo mi ropa la noche anterior a una entrevista para que no se me haga tarde» —ítem cuya puntuación se invierte-). El formato de respuesta de la escala es tipo Likert con cinco opciones de respuesta (que van desde el 1 — «no me describe en absoluto»— hasta el 5 - «muy característico»). Todos los valores del alfa de Cronbach obtenidos en el presente estudio fueron aceptables $\left(\alpha_{\mathrm{GP}}=.79 ; \alpha_{\mathrm{DP}}=\right.$ $\left..88 ; \alpha_{\mathrm{AIP}}=.87\right)$.

\subsection{Procedimiento de recogida de datos}

El estudio siguió los requerimientos de la Comisión de Bioética de la Universidad de Barcelona (CBUB, IRB00003099), no siendo necesarias más aprobaciones dado que los datos obtenidos no implicaban experimentación animal o clínica. Esta investigación, además, cumple con las recomendaciones del Consejo General de la Psicología de España, la Ley Orgánica Española de Protección de Datos (15/1999: Jefatura del Estado, 1999) y la Declaración de Helsinki (World Medical Association, 2013).

El trabajo de campo fue precedido de dos fases de preparación, siguiendo las pautas de investigaciones anteriores desarrolladas en el ámbito de las actividades de ocio (Codina y Pestana, 2017; Codina, Pestana y Stebbins, 2017; Codina, Pestana, Romeo y Yepes, 2019) que utilizaron la modalidad de panel de participantes. En la primera fase, el equipo investigador trabajó con el personal técnico especializado para que introdujera los ítems en el software con el formato que visualizarían los participantes. Con la finalidad de evitar pérdidas de información, el cuestionario se programó de manera que, para cumplimentarlo, fuera necesario contestar a cada una de sus preguntas. Las categorías de respuesta de cada pregunta eran visibles en una misma pantalla, para evitar tener que hacer desplazamientos por la misma. Verificada la última programación del cuestionario, se inició la segunda fase con una prueba piloto. A partir de esta prueba se practicaron los ajustes de formato necesarios.

Tras unas últimas comprobaciones de funcionamiento, a los potenciales participantes del panel se les envió un mensaje de correo electrónico invitándoles a formar parte del estudio, con un enlace directo al instrumento (vínculo único y al que no se podía volver una vez enviadas las respuestas). El acceso a las preguntas se estableció durante el mes de noviembre de 2019. La invitación se dirigió únicamente a quienes, del panel de potenciales participantes, cumplían con el requisito de edad establecido. 
2.4. Procedimiento de análisis de la información

Los datos obtenidos se analizaron con el programa SPSS en su versión 25. Tras los estadísticos descriptivos (frecuencias y porcentajes para las variables sociodemográficas; medias, desviaciones típicas, asimetría, curtosis y homocedasticidad para la perspectiva temporal y la procrastinación), las asociaciones entre las variables se han calculado mediante comparación de medias (con la $t$ de Student o ANOVAs - según procede). En el caso de las asociaciones significativas, se precisa en el texto el tamaño del efecto.

\section{Resultados}

\subsection{Datos sociodemográficos}

De la información sociodemográfica obtenida (Tabla 1), la edad fue analizada según los tres grupos de edad siguientes: $18-29(n=128 ; 17.8 \%), 30-49(n=417$; $57.9 \%)$ y $50-64(n=175 ; 24.3 \%)$.

TABLA 1. Prevalencia de las variables sociodemográficas: sexo, edad y cohabitación ( $N=720)$.

\begin{tabular}{|c|c|c|}
\hline Variables & $\mathbf{n}$ & $\%$ \\
\hline \multicolumn{3}{|l|}{ Sexo } \\
\hline Hombre & 390 & 54.2 \\
\hline Mujer & 330 & 45.8 \\
\hline \multicolumn{3}{|l|}{ Edad } \\
\hline $18-29$ & 128 & 17.8 \\
\hline $30-49$ & 417 & 57.9 \\
\hline $50-64$ & 175 & 24.3 \\
\hline \multicolumn{3}{|l|}{ Cohabitación } \\
\hline \multicolumn{3}{|l|}{ Solo } \\
\hline Sí & 101 & 14 \\
\hline No & 619 & 86 \\
\hline \multicolumn{3}{|l|}{ Pareja } \\
\hline Sí & 452 & 62.8 \\
\hline No & 268 & 37.2 \\
\hline \multicolumn{3}{|l|}{ Hijos } \\
\hline Sí & 299 & 41.5 \\
\hline No & 421 & 58.2 \\
\hline \multicolumn{3}{|l|}{ Padres } \\
\hline Sí & 53 & 7.4 \\
\hline No & 667 & 92.6 \\
\hline \multicolumn{3}{|c|}{ Personas a cargo } \\
\hline Sí & 37 & 6 \\
\hline No & 683 & 94 \\
\hline
\end{tabular}

Fuente: Elaboración propia. 
Con respecto a las circunstancias de cohabitación (Tabla 1), en el conjunto de la muestra 101 participantes vivían solas/os (14\%), 452 en pareja (62.8\%), 299 con hijas/os $(41.5 \%), 53$ con los padres $(7.4 \%)$ y 37 tenían personas a su cargo (un $6 \%$ del total).

De las variables relativas a las características temporales del trabajo (Tabla infrecuente.
2), predominan quienes destinan como máximo media hora para desplazarse al lugar de trabajo $(n=524 ; 72.8 \%)$, trabajan 40 horas y más a la semana $(n=417$; $57.9 \%)$, tienen un horario laboral rígido $(n=330 ; 45.8 \%)$ y no suelen prolongar su jornada laboral $(n=240,33.33 \%)-$ si bien el $18.6 \%$ de los participantes han prolongado la jornada laboral de manera

TABLA 2. Prevalencia de las variables sociodemográficas: organización temporal del trabajo $(\mathrm{N}=720)$.

\begin{tabular}{|ccc|}
\hline Variables & n & $\%$ \\
\hline Tiempo desplazamientos & 524 & 72.8 \\
Máx. 30' & 156 & 21.7 \\
Máx. 60' & 40 & 5.6 \\
Más de 60' & & \\
\hline Horas trabajadas & 303 & 42.1 \\
Hasta 39 h & 417 & 57.9 \\
\hline 40 h y más & & \\
Flexibilidad horaria & 330 & 45.8 \\
Rígido & 239 & 33.2 \\
Flexible (trabajador/a) & 151 & 21 \\
\hline Flexible (empresa) & & 14 \\
(Casi) cada día & 101 & 17.8 \\
Más de un día / semana & 128 & 16.3 \\
Al menos una vez al mes & 117 & 18.6 \\
Infrecuente & 134 & 33.33 \\
No & 240 & \\
\hline
\end{tabular}

Fuente: Elaboración propia.

\subsection{Perspectiva temporal}

De las cinco dimensiones de la perspectiva temporal (Tabla 3), los valores mas elevados se observan en los casos del futuro $(M=$ $3.49 ; D T=0.42)$ y el pasado positivo $(M=$ $3.42 ; D T=0.46)$. En cambio, los valores más bajos correspondieron al presente fatalista $(M=2.88 ; D T=0.57)$. Por lo que respecta a los valores de la asimetría, la curtosis y el test de Kolmogorov-Smirnoff, en todas las dimensiones de la perspectiva temporal se comprueba la no normalidad de la muestra. 
TABLA 3. Medias, desviaciones típicas, asimetría, curtosis y test de KolmogorovSmirnov (K-S) para las perspectivas temporales y los factores de la procrastinación.

\begin{tabular}{|lcccccccc|}
\hline & \multicolumn{9}{c}{ Asimetría } & \multicolumn{2}{c}{ Curtosis } & \multicolumn{2}{c|}{ K-S } \\
& M & DT & Valor & EE & Valor & EE & Valor & p \\
\hline Perspectivas temporales & & & & & & & & \\
$\quad$ Pasado negativo & 2.98 & 0.62 & .094 & .091 & .349 & .182 & .085 & .000 \\
$\quad$ Pasado positivo & 3.42 & 0.46 & -.105 & .091 & .524 & .182 & .055 & .000 \\
$\quad$ Presente hedonista & 3.20 & 0.52 & .085 & .091 & .978 & .182 & .063 & .000 \\
$\quad$ Presente fatalista & 2.88 & 0.57 & .321 & .091 & .687 & .182 & .081 & .000 \\
$\quad$ Futuro & 3.49 & 0.42 & -.075 & .091 & .371 & .182 & .046 & .001 \\
\hline Factores de la & & & & & & & & \\
procrastinación & & & & & & & & \\
$\quad$ Conductas dilatorias & 2.56 & 0.65 & .176 & .091 & .170 & .182 & .051 & .000 \\
$\quad$ Indecisión & 2.58 & 0.68 & .066 & .091 & -.008 & .182 & .072 & .000 \\
$\quad$ Falta de puntualidad & 2.28 & 0.71 & .211 & .091 & -.485 & .182 & .079 & .000 \\
$\quad$ Falta de planificación & 2.26 & 0.41 & .163 & .091 & 1.118 & .182 & .063 & .000 \\
\hline
\end{tabular}

Fuente: Elaboración propia.

$\mathrm{Al}$ asociar estos valores con los datos sociodemográficos, varias de las dimensiones de la perspectiva temporal presentaron diferencias significativas de acuerdo con el sexo, la edad, las circunstancias de cohabitación y la organización temporal alrededor del entorno laboral (Tablas 4 y 5), si bien con distintos tamaños del efecto.

Al comparar mujeres y hombres (Tabla 4), en ellas se evidenciaron mayores valores de presente fatalista $(t=-2.43 ; p<.015 ; d=.17)$, aunque con un efecto pequeño. Con respecto a la edad, en los más jóvenes se observan los mayores valores en el pasado negativo $(F=7.67$; $p<.001 ; d=.61)$, el presente hedonista $(F=$ $7.70 ; p<.000 ; d=.51)$ y el presente fatalista $(F=5.703 ; p<.003 ; d=.59)$-en todos los casos, con un tamaño del efecto grande.

Por lo que se refiere a las circunstancias de cohabitación (Tabla 4), en las personas que viven solas hubo mayor presente fatalista $(t=-2.08 ; p<.039 ; d=.20)$ y menor futuro $(t=2.41 ; p<.016 ; d=.27)$-en ambos casos, con un efecto pequeño. Entre las personas con hijas/os se observó - con un efecto pequeño- menor presente hedonista $(t=2.31 ; p<.021 ; d=.17)$ y presente fatalista $(t=2.35 ; p<.019 ; d=.19)$. Por su parte, quienes viven con sus padres presentan - de manera significativa y con un efecto moderado - más pasado negativo $(t=2.35 ; p<.019 ; d=.33)$ y presente hedonista $(t=-2.16 ; p<.031 ; d=.30)$; en cambio, quienes viven con personas a su cargo, presentan un presente fatalista más acentuado $(t=2.29 ; p<.027 ; d=.22)$, si bien el efecto es pequeño.

Finalmente, cuatro aspectos del entorno laboral influyeron en dimensiones de la perspectiva temporal (Tabla 5) -en todos los casos con un tamaño del efecto grande. 


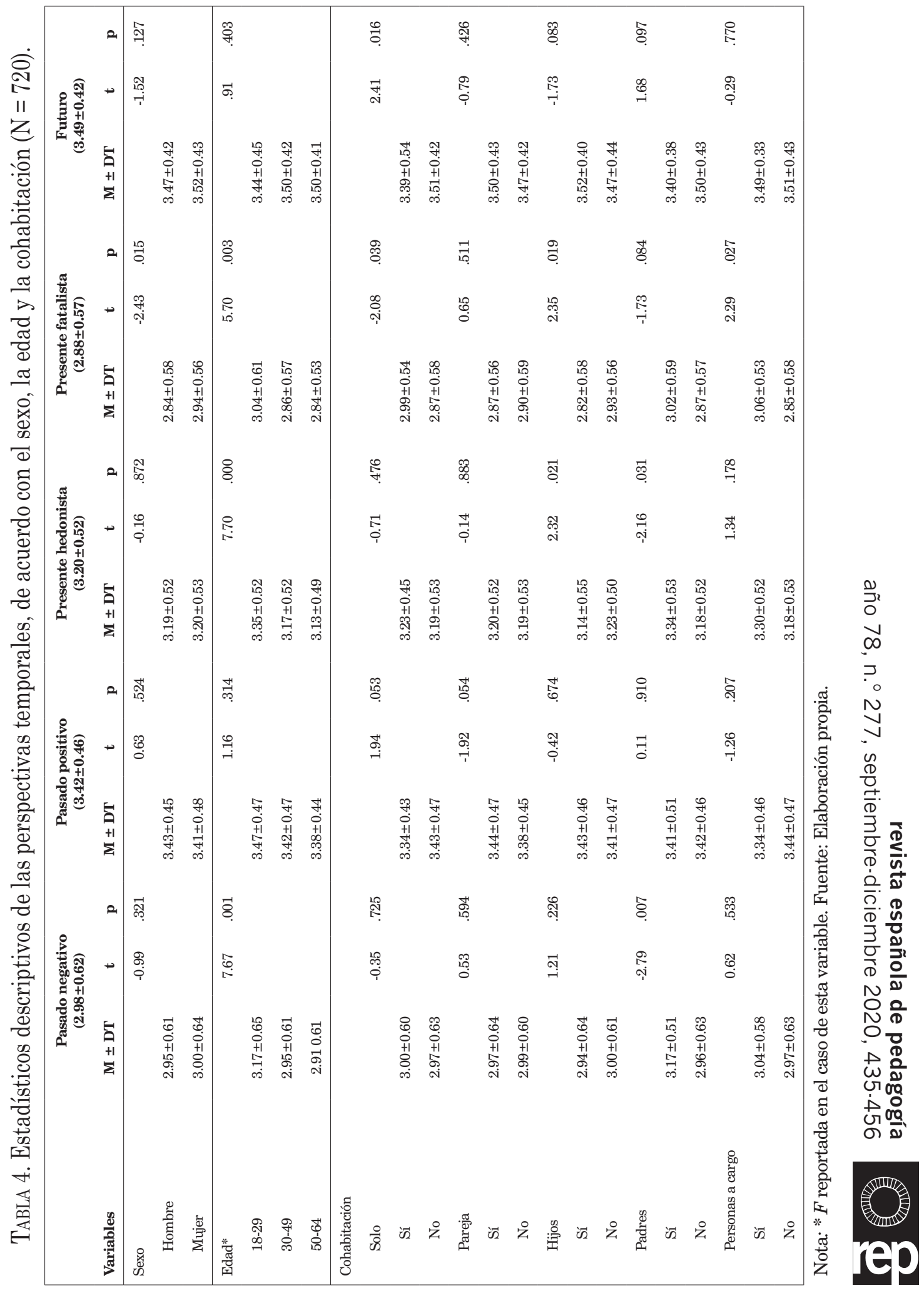




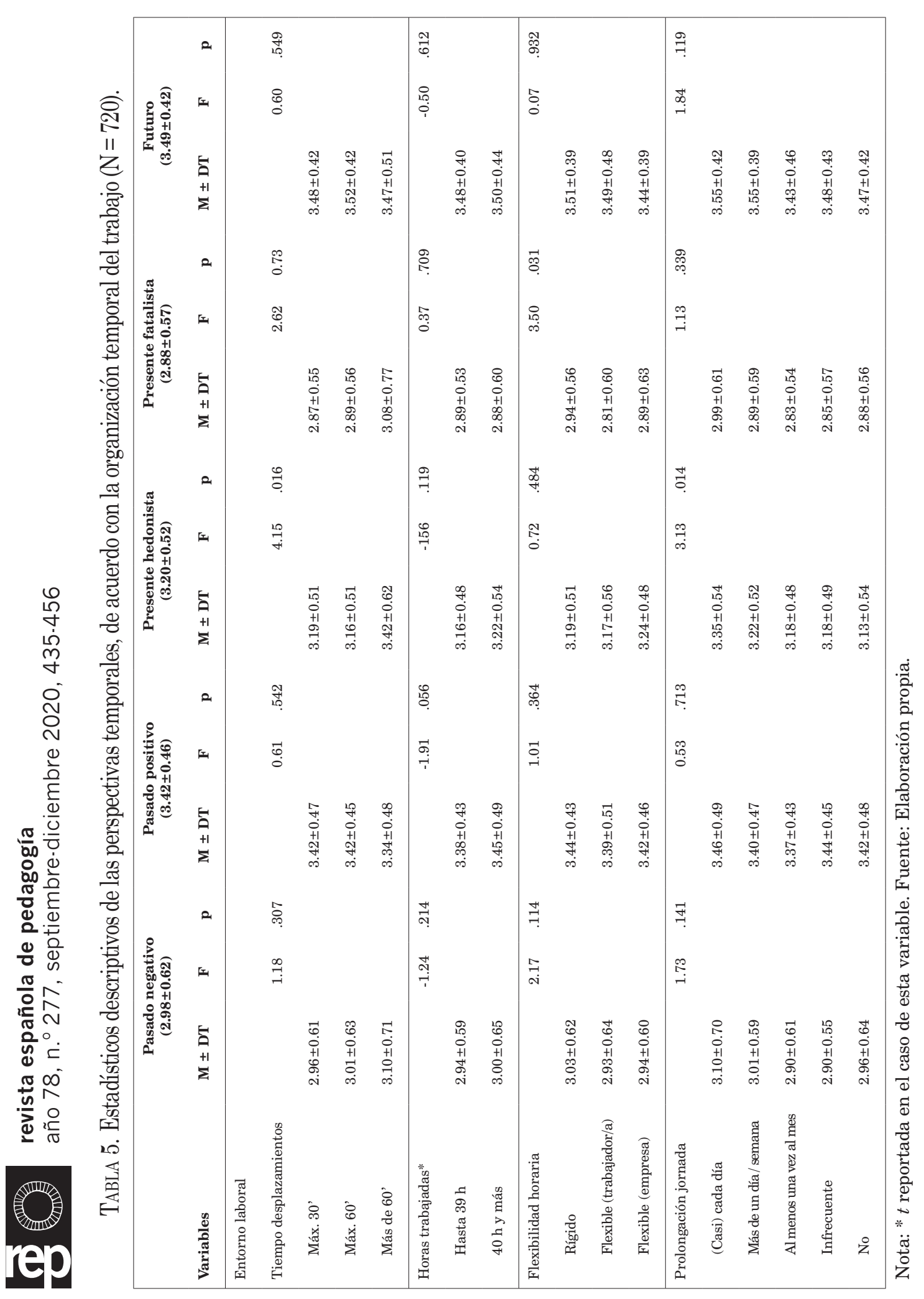


Quienes invierten entre más de una hora en desplazarse al trabajo muestran mayor presente hedonista $(F=4.15 ; p$ $<.016 ; d=.51$ ). Cuando los horarios de trabajo son rígidos se observa más presente fatalista $(F=3.50 ; p<.031$; $d=.53)$. Y cuando la jornada laboral se alarga cada día - 0 casi- hay un mayor presente hedonista $(F=3.13 ; p<.014$; $d=.51)$.

\subsection{Procrastinación}

En el conjunto de la muestra, de los cuatro factores de procrastinación (Tabla 3), obtuvieron las mayores puntuaciones la indecisión $(M=2.58 ; D T=$ $0.68)$ y los comportamientos dilatorios $(M=2.56 ; D T=0.65)$-ambos con valores similares-. Consecuentemente, el llegar tarde $(M=2.28 ; D T=0.71) \mathrm{y}$ la falta de planificación $(M=2.26 ; D T$ $=0.41$ ) fueron los factores con menor presencia (igualmente con puntuaciones semejantes entre ambos). En relación con los valores de la asimetría, la curtosis y el test de Kolmogorov-Smirnoff, todos los factores de la procrastinación se distribuyen de manera no normal en el conjunto de la muestra.

$\mathrm{Al}$ asociar las variables sociodemográficas con los factores de la procrastinación, hubo diferencias significativas según: la edad, vivir solo / con hijos y la prolongación de la jornada laboral (Tablas 6 y 7 ).

En el caso del sexo, no se observaron diferencias significativas en la procrastinación de hombres y mujeres. En cambio, los participantes entre 18-29 años presentaron las mayores puntuaciones en todos los factores de este problema de dominio o control del tiempo (Tabla 6), a excepción del factor de indecisión. En concreto, los valores significativos obtenidos fueron los siguientes: conductas dilatorias $(F=7.67 ; p<.001 ; d=$ .63 ), falta de puntualidad ( $F=7.70 ; p$ $<.000 ; d=.69)$ y falta de planificación $(F=5.70 ; p<.003 ; d=.40)$, siendo grandes los tamaños del efecto en los factores de conductas dilatorias y falta de puntualidad, y moderado en el caso de la falta de planificación.

Por lo que respecta a la forma de cohabitación de las/os participantes (Tabla 6), el vivir solo se caracteriza por mayor falta de planificación $(t=-3.09$; $p<.002 ; d=.34)$, siendo moderado el tamaño del efecto; en contraposición, la falta de planificación es mayor cuando se vive en pareja $(t=2.45 ; p<.014 ; d$ $=.19)$, aún cuando el tamaño del efecto es pequeño. En relación a cohabitar con hijos -y si bien los tamaños del efecto también son pequeños- esta circunstancia se caracteriza por una mayor presencia de comportamientos dilatorios $(t$ $=2.41 ; p<.016 ; d=.18)$, indecisión $(t$ $=2.64 ; p<.008 ; d=.20)$ y falta de puntualidad ( $t=2.33 ; p<.020 ; d=.18)$.

De las variables relativas a la organización temporal del trabajo (Tabla 7), quienes prolongan la jornada laboral cada día - 0 casi cada día-presentan más comportamientos dilatorios $(F$ $=3.15 ; p<.014 ; d=.64$ ), asociación significativa cuyo tamaño del efecto es grande. 


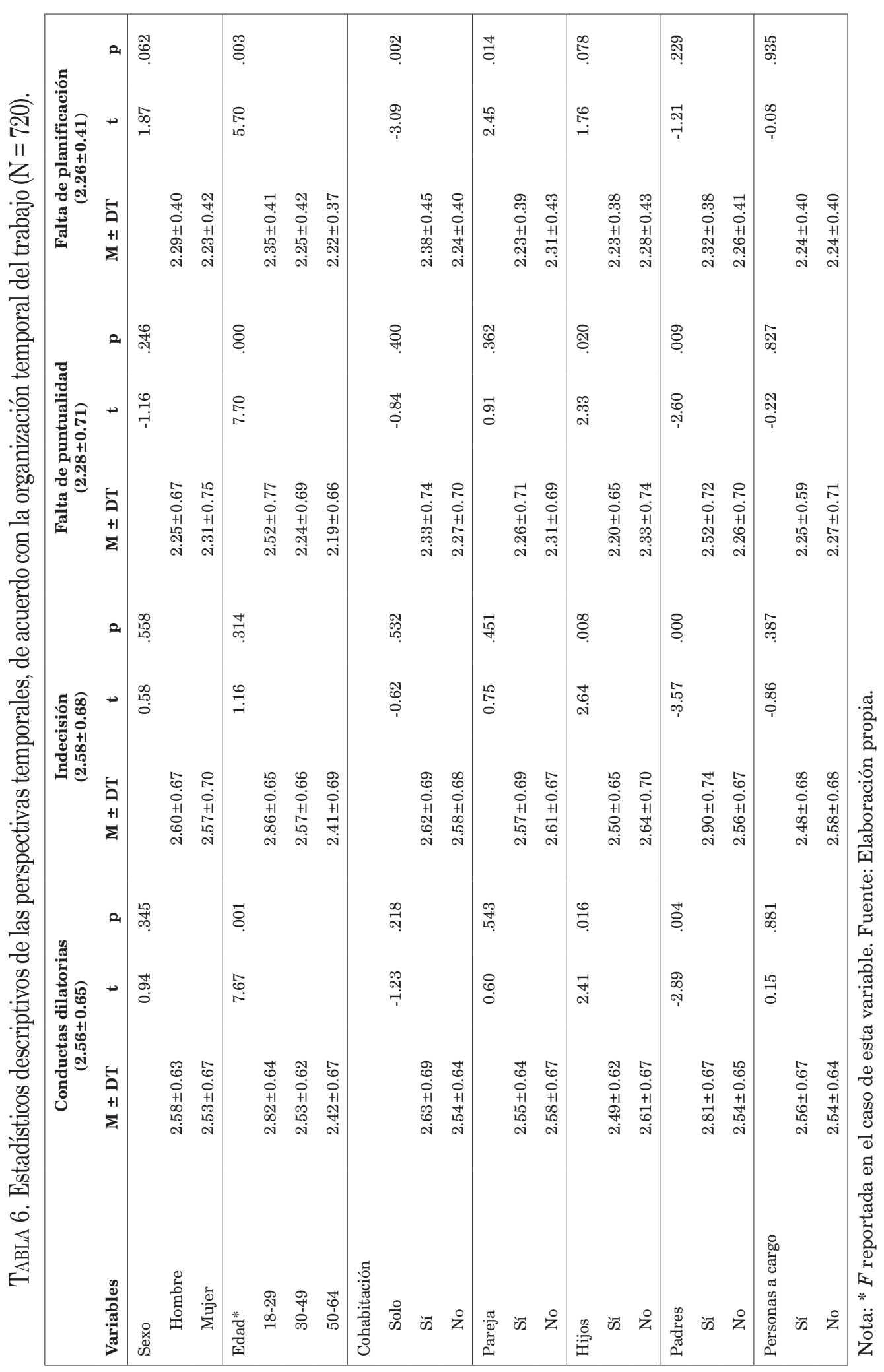




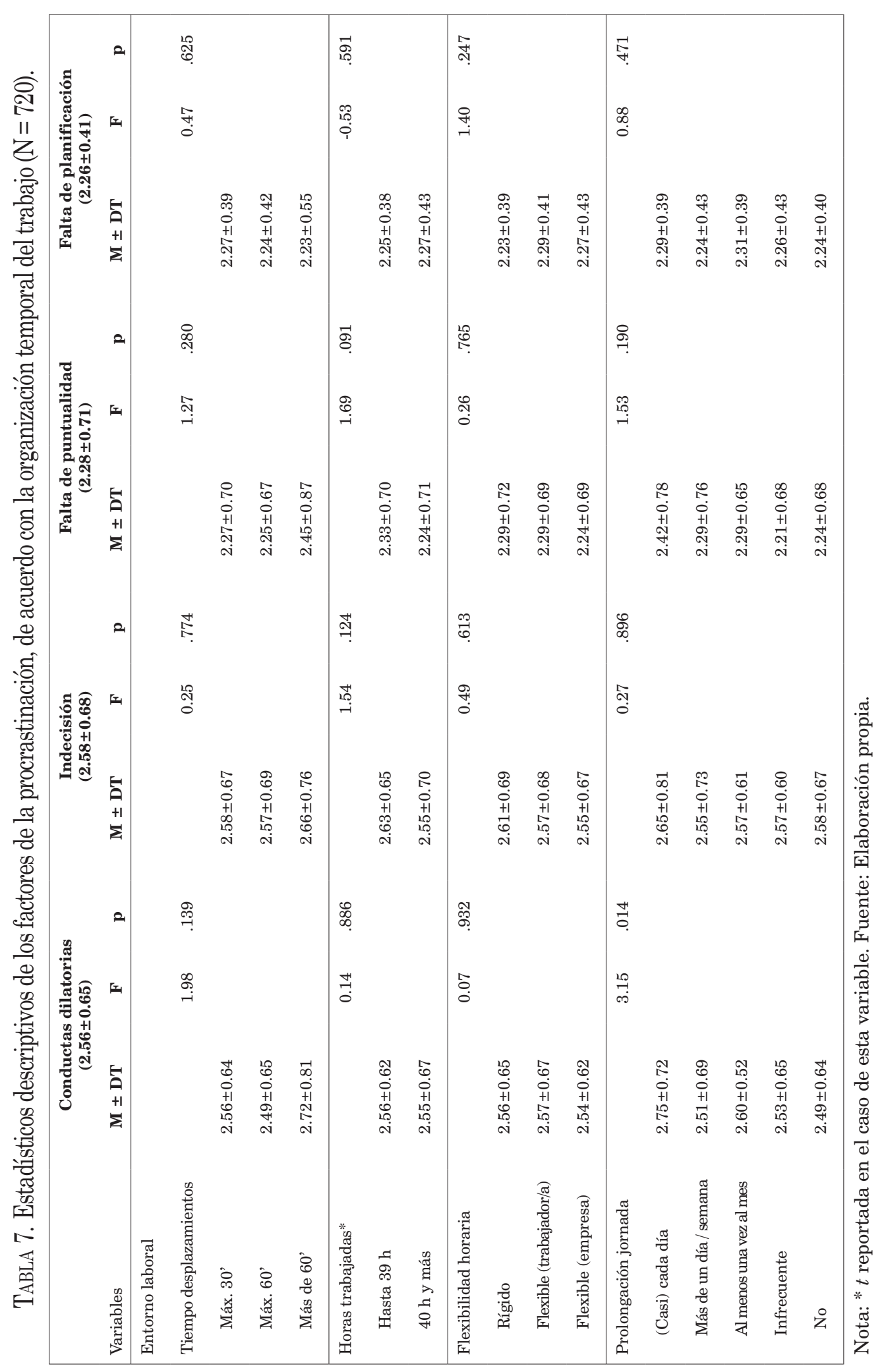

อั้ $\searrow^{\infty}$

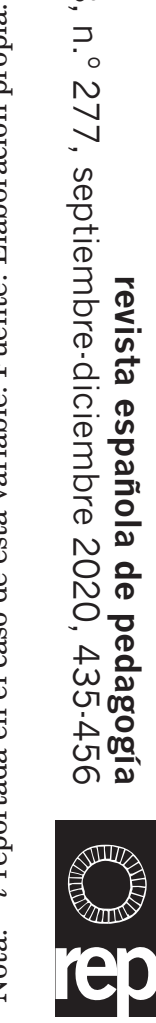




\section{Discusión}

En esta investigación, al estudiar las perspectivas temporales y la procrastinación en relación con variables personales y situacionales como la edad, la cohabitación y las características temporales del trabajo se aporta información novedosa que enriquece los conocimientos de ambos conceptos - los cuales pueden ser aplicados tanto en investigación como en intervención.

Respecto a la dinámica de perspectivas temporales que configuran una orientación temporal equilibrada (Boniwell y Zimbardo, 2004; Sircova et al., 2014, entre otros), se puede afirmar que la muestra estudiada no la presenta. No obstante, en general -y de manera bastante estable en las diferentes franjas de edad- la muestra puntúa alto en las dos perspectivas funcionales (la de pasado positivo y futuro) y puntúa bajo en una de las dos disfuncionales (la de presente fatalista). Por lo tanto, aún no cumpliendo con el estándar del perfil de una perspectiva temporal equilibrada u óptima, sí que se acerca. En un sentido más práctico, en la muestra estudiada, la competencia temporal (Zaleski, 1994), para adaptarse de manera hábil y flexible frente a retos de la vida, se ve afectada negativamente por cierta incidencia del pasado negativo.

Frente a estos resultados generales, los jóvenes trabajadores - de entre 18 y 29 años de edad - presentan un perfil de perspectivas temporales más inquietante: puntúan alto en las dos perspectivas temporales disfuncionales - la de pasado negativo y presente fatalista - así como en la de presente hedonista. De acuerdo con los estudios previos (Ferrari y Díaz-Morales,
2007 o Shell y Husman, 2001), el puntaje alto en las dos perspectivas de presente altas indica que se sienten bien y seguros en diferentes entornos. Ahora bien, llama la atención que entre los jóvenes trabajadores no destaque un predominio de la perspectiva de futuro, un dato que hace pensar en que - muy probablemente y a modo de compensación frente a un futuro incierto- se intensifique el presente hedonista.

Por lo que se refiere a una de la variables sociodemográficas no estudiadas previamente en relación con la perspectiva temporal, la situación de cohabitación, los datos obtenidos revelan realidades insospechadas. En efecto, se muestra cuán potente es la convivencia en las perspectivas temporales personales. En concreto, se observa que las personas que viven solas puntúan alto en la perspectiva de presente fatalista y bajo en la de futuro - por lo que parecen tener unas actitudes temporales poco positivas y estimulantes; un dato que lleva a advertir la relevancia de convivir con otras personas, cuestión que será necesario explorar con más detalle en un futuro. Por lo que se refiere a las personas que conviven con otras generaciones, se ha visto que: la presencia de hijos en el hogar inhibe las perspectivas temporales de presente (tanto fatalista como hedonista), posiblemente por las novedades, incertezas y cambios vinculados a la vida de los hijos; vivir con los padres activa las perspectivas de pasado negativo y presente hedonista, como si se tratara de una compensación entre la actitud hacia un pasado no satisfactorio y el vivir el presente de la mejor manera; y, finalmente, convivir con personas a cargo se asocia con un predominio de la perspectiva de presente 
fatalista - en este caso, las condiciones de la convivencia-dependencia posiblemente son vividas como un factor limitador para vivir el presente 0 hacer planes de futuro-. Por lo tanto, la convivencia con personas de otras generaciones condiciona claramente y de manera diferenciada — según el rol que se desempeñe dentro del hogar- las perspectivas temporales predominantes.

Los resultados obtenidos sugieren que las intervenciones psicoeducativas, orientadas a las buenas prácticas en el dominio o control del tiempo, deben atender a las realidades diferenciadas que las situaciones de cohabitación promueven en los diferentes individuos. Por ejemplo, si bien alguien con personas a su cargo puede tener mermadas sus actitudes hacia el futuro, esta característica debería modificarse una vez cambia esta situación de responsabilidad ante alguien dependiente (sobre las intervenciones con cuidadores de personas dependientes, véase Yuan y Jiang, 2017).

Por lo que se refiere a las dinámicas entre las perspectivas temporales y características temporales del trabajo, también hemos identificado algunas actitudes temporales negativas y positivas insospechadas. Respecto a las negativas, si la jornada de trabajo tiene una estructura horaria rígida, se puntúa alto en la perspectiva de presente fatalista; por lo tanto, en convergencia con los resultados obtenidos por Cladellas y Badia (2010) respecto a la gestión del tiempo del profesorado, tener unos horarios de trabajo fijos y establecidos por otras personas es disfuncional. Por lo que se refiere a las relaciones positivas, si ir al trabajo requiere de más de 60 minutos de desplazamiento o si la jornada de trabajo se alarga, se puntúa más alto en la perspectiva de presente hedonista; por lo tanto, este tiempo de desplazamiento (que no es trabajo ni es ocio), lejos de suponer una carga negativa, parece aportar optimismo en las actitudes temporales - ocurriendo de manera similar con la prolongación de la jornada de trabajo- De resultas de estos datos, aparecen la flexibilidad de la jornada de trabajo y el disfrute del tiempo de desplazamiento hacia este como dos elementos a tener en cuenta en el fomento de la experiencia de control del tiempo en quienes trabajan - lo que, a la larga, podría constituir una fuente de bienestar, calidad de vida 0 ambos (como señalan Yang, Xu y Zhu, 2015).

Por su parte, la procrastinación es un problema que, en líneas generales, no se da en todas sus dimensiones en la muestra estudiada. En particular, no son relevantes sus manifestaciones de llegadas tarde o no planificación; por el contrario, sí que se observan conductas procrastinadoras respecto a la indecisión y dilación. Ahora bien, cuando se valora la procrastinación por franjas de edad, se descubre que los jóvenes de 18 a 29 años puntúan significativamente alto en casi todos los factores de procrastinación (a excepción de la indecisión). En consecuencia, entre las personas trabajadoras -que no universitarios - quienes suelen procrastinar más son los jóvenes, tendencia documentada sobre todo en el ámbito académico (van Eerde, 2003). Esta constatación respecto a los jóvenes trabajadores procrastinadores, junto con el hallazgo relativo al predominio de las perspectivas temporales de presente - además de apoyar estudios previos en los que la falta de futuro correlaciona con la procrasti- 
nación (Díaz-Morales, 2019)— ponen sobre aviso la necesidad de investigar con detalle el colectivo de jóvenes trabajadores.

Respecto a las condiciones de convivencia y procrastinación, se ha encontrado que quienes viven solos planifican menos; en este sentido, parece que la no necesidad de coordinación con otros hace descuidar este aspecto de gestión del tiempo -recuérdese que los que viven solos también son quienes tienen menos perspectiva de futuro-. Por el contrario, quienes viven en pareja puntúan más alto en planificación. Otro dato de interés es valorar las tendencias procrastinadoras entre quienes viven con los hijos; en este caso, son bajas las puntuaciones de comportamientos dilatorios, indecisión y llegadas tarde; resultados que parecen responder al rol de la persona que cuida, educa y protege a sus descendientes. Como puede observarse, en las intervenciones orientadas a favorecer el control 0 dominio del tiempo - contraviniendo los efectos de la procrastinación - también es importante atender a las circunstancias de cohabitación. Piénsese, por ejemplo, en los cambios que una persona puede experimentar cuando deja de ser el principal responsable de la rutina de sus descendientes.

Los resultados obtenidos - reveladores y, al tiempo, prometedores- no obvian las limitaciones del estudio. A este respecto, considerar futuras muestras que, además de estar distribuidas según el sexo y la edad, incorporen otras variables (como distribución territorial 0 actividades distintas al trabajo), pueden fortalecer las asociaciones obtenidas en perspectiva temporal y procrastinación (y sus correspondientes tamaños del efecto). Otro aspecto a tener en cuenta en futuras investigaciones es el tipo de muestreo utilizado; así, a diferencia de una muestra intencional en un panel, cabe considerar una muestra aleatoria sobre la propia base del padrón municipal.

En resumen, este trabajo pone de relieve cómo las condiciones de convivencia y ciertas características temporales del trabajo están asociadas al perfil de perspectivas temporales, así como las tendencias procrastinadoras. Estos hallazgos, pues, dan a conocer condiciones laborales y de cohabitación - sobre las que los agentes de intervención pueden incidir- que se relacionan positiva y negativamente en el dominio del tiempo, constituyendo evidencias respecto a variables que no pueden dejar de contemplar futuras investigaciones.

\section{Referencias bibliográficas}

Ato, M., López, J. J. y Benavente, A. (2013). Un sistema de clasificación de los diseños de investigación en psicología. Anales de Psicología, 29 (3), 1038-1059. doi: https://doi.org/10.6018/ analesps.29.3.178511

Bluedorn, A. C. (2002). The human organization of time: Temporal realities and experience. Redwood City, CA: Stanford University Press.

Boniwell, I. y Zimbardo, P. G. (2004). Balancing time perspective in pursuit of optimal functioning. En P. A. Linley y S. Joseph (Eds.), Positive psychology in practice (pp. 165-178). Hoboken, NJ: Wiley.

Boniwell, I., Osin, E., Linley, P. A. e Ivanchenko, G. V. (2010). A question of balance: Time perspective and well-being in British and Russian samples. Journal of Positive Psychology, 5, 24-40. doi: https://doi.org/10.1080/17439760903271181

Cladellas, R. y Badia, M. (2010). La gestión del tiempo de los profesores universitarios en función de la modalidad educativa: sus efectos psi- 
cosociales. revista española de pedagogía, 68 (246), 297-310.

Codina, N. y Pestana, J. V. (2016). Actividad físicodeportiva como experiencia de ocio y Perspectiva Temporal en los jóvenes. Revista de Psicología del Deporte, 25 (Suppl. 2), 53-60.

Codina, N. y Pestana, J. V. (2017). Two sides of time in the leisure experience of youth: Time investment and time perspectives. Loisir et Société / Society and Leisure, 40 (3), 310-323. doi: 10.1080/07053436.2017.1378508

Codina, N., Castillo, I., Pestana, J. V. y Balaguer, I. (2020). Preventing Procrastination Behaviours: Teaching Styles and Competence in University Students. Sustainability, 12, 2448. doi: https://doi.org/10.3390/su12062448

Codina, N., Pestana, J. V. y Ponce de León Elizondo, A. (2018). Tiempos dedicados al ocio físico-deportivo y perspectivas temporales: (Re)velando vulnerabilidades. Pedagogía Social. Revista Interuniversitaria, 31, 59-69. doi: 10.SE7179/PSRI_2018.31.05

Codina, N., Pestana, J. V. y Stebbins, R. A. (2017). Serious and casual leisure activities in the construction of young adult identity: a study based on participants' self-descriptions. OBETS. Revista de Ciencias Sociales, 12 (Extra 1), 65-80. doi: http://dx.doi.org/10.14198/ OBETS2017.12.1.12

Codina, N., Pestana, J. V., Romeo, M. y YepesBaldo, M. (2019). When shopping and leisure go together in malls. User experiences in Barcelona. Anuario de Psicología. The UB Journal of Psychology, 49 (1), 49-56. doi: https://doi. org/10.1344/anpsic2019.49.6

Codina, N., Valenzuela, R., Pestana, J. V. y González-Conde, J. (2018). Relations Between Student Procrastination and Teaching Styles: Autonomy-Supportive and Controlling. Frontiers in Psychology, 9, 809. doi: https://doi. org/10.3389/fpsyg.2018.00809

D’Alessio, M., Guarino, A., De Pascalis, V. y Zimbardo, P. G. (2003). Testing Zimbardo's Stanford Time Perspective Inventory (STPI) - Short Form: An Italian study. Time and Society, 12 (2-3), 333-347. doi: https://doi.or$\mathrm{g} / 10.1177 / 0961463 X 030122010$

Díaz-Morales, J. F. (2006). Estructura factorial y fiabilidad del Inventario de Perspectiva Temporal de Zimbardo. Psicothema, 18 (3), 565-571.
Díaz-Morales, J. F. (2019). Procrastination. A review of scales and correlates. Revista Iberoamericana de Diagnóstico y Evaluación - e Avaliação Psicológica. RIDEP, 51 (2), 43-60. doi: https://doi.org/10.21865/RIDEP51.2.04

Díaz-Morales, J. F. y Ferrari, J. R. (2015). More time for procrastinators: The role of time perspective (pp. 305-321). En M. Stolarski, N. Fieulaine y W. van Beek (Eds.), Time perspective theory: Review, research and application. Londres: Springer.

Díaz-Morales, J. F., Ferrari, J. R., Díaz, K. y Argumedo, D. (2006). Procrastination and demographic characteristics in Spanish adults: Further evidence. The Journal of Social Psychology, 146, 629-633. doi: https://doi.org/10.3200/ SOCP.146.5.629-633

Drake, L., Duncan, E., Sutherland, F., Abernethy, C. y Henry, C. (2008). Time Perspective and correlates of wellbeing. Time and Society, 17, 47-61. doi: https://doi.org/10.1177/0961463X07086304

Ferrari, J. R. y Díaz-Morales, J. F. (2007). Procrastination: Different time orientations reflect different motives. Journal of Research in Personality, 41, 707-714. doi: https://doi.org/10.1016/j.jrp.2006.06.006

Goroshit, M. (2018). Academic procrastination and academic performance: an initial basis for intervention. Journal of Prevention \& Intervention Community, 46 (2), 131-142. doi: https:// doi.org/10.1080/10852352.2016.1198157

Harriott, J. y Ferrari, J. R. (1996). Prevalence of procrastination among samples of adults. Psychological Reports, 78 (2), 611-616. doi: https:// doi.org/10.2466/pr0.1996.78.2.611

Kim, S., Fernández, S. y Terrier, L. (2017). Procrastination, personality traits, and academic performance: when active and passive procrastination tell a different story. Personality and Individual Differences, 108, 154-157. doi: https://doi.org/10.1016/j.paid.2016.12.021

Klingsieck, K. B. (2013). Procrastination: When good things don't come to those who wait. $\mathrm{Eu}$ ropean Psychologist, 18, 24-31. doi: https://doi. org/10.1027/1016-9040/a000138

Kooij, D. T., Kanfer, R., Betts, M. y Rudolph, C. W. (2018). Future time perspective: A systematic review and meta-analysis. Journal of Applied Psychology, 103, 867-893. doi: https://doi. org/10.1037/apl0000306 
Laureiro-Martínez, D., Trujillo, C. A. y Unda, J. (2017). Time perspective and age: A review of age associated differences. Frontiers in Psychology, 8, 101. doi: https://doi.org/10.3389/ fpsyg.2017.00101

Lay, C. H. (1986). At last, my research article on procrastination. Journal of Research in Personality, 20, 474-495. doi: 10.1016/00926566(86)90127-3

Levasseur, L., Shipp, A., Fried, Y., Rousseau, D. y Zimbardo, F. (2020). New perspectives on time perspective and temporal focus. Journal of Organizational Behavior, 41 (3), 235-243. doi: https://doi.org/10.1002/job.2435

Lewin, K. (1948). Resolving social conflicts. Selected papers on group dynamics. Field theory in social science. Nueva York, NY: Harper \& Brothers.

Ley Orgánica 15/1999, de 13 de diciembre, de Protección de Datos de Carácter Personal. Boletin Oficial del Estado, 298, de 14 de diciembre de 1999, páginas 4308843099. Recuperado de https://www.boe.es/ boe/dias/1999/12/14/pdfs/A43088-43099.pdf (Consultado el 15-03-2020).

Mann, L. (1982). Decision Making Questionnaire: Parts I and II. En J. R. Ferrari, J. L. Johnson y W. McCown (Eds.), Procrastination and task avoidance. Nueva York, NY: Plenum Press.

Matthews, G. y Stolarski, M. (2015). Emotional processes in development and dynamics of individual time perspective. En M. Stolarski, N. Fieulaine y W. van Beek (Eds.), Time perspective theory: Review, research and application (pp. 269-286). Cham, Suiza: Springer International Publishing.

McCown, W., Johnson, J. y Petzel, T. (1989). Procrastination, a principal components analysis. Personality and Individual Differences, 10, 197-202. doi: https://doi.org/10.1016/01918869(89)90204-3

Nuttin, J. (1985). Future time perspective and motivation: Theory and research method. Lovaina, Bélgica: Leuven University Press y Lawrence Erlbaum Associates.

Pestana, J. V., Codina, N. y Valenzuela, R. (2020). Leisure and Procrastination, a Quest for Autonomy in Free Time Investments: Task
Avoidance or Accomplishment? Frontiers in Psychology, 10, 2918. doi: https://doi. org/10.3389/fpsyg.2019.02918

Shell, D. F., y Husman, J. (2001). The multivariate dimensionality of personal control and future time perspective beliefs in achievement and self-regulation. Contemporary Educational Psychology, 26 (4), 481-506. doi: https://doi.org/10.1006/ceps.2000.1073

Simons, M., Peeters, S., Janssens, M., Lataster, J. y Jacobs, N. (2018) Does age make a difference? Age as moderator in the association between time perspective and happiness. Journal of Happiness Studies, 19, 57-67 doi: https://doi.org/10.1007/s10902-016-9806-1

Sircova, A., van de Vijver, F. J. R., Osin, E., Milfont, T. L., Fieulaine, N., Kislali Erginbilgic, A., ... Boyd, J. N. (2014). A global look at time: A 24 country study of the equivalence of the Zimbardo Time Perspective Inventory. SAGE Open, 4 (1), 1-12. doi: https://doi. org/ $10.1177 / 2158244013515686$

Soylu, C. y Ozekes, B. C. (2019). Psychometric Properties of the Future Time Perspective Scale for the Turkish Population: Age Differences in Predictors of Time Perspective. The International Journal of Aging and Human Development, 91 (1), 85-106. doi: https://doi. org $/ 10.1177 / 0091415019836108$

Steel, P. (2007). The nature of procrastination: A meta-analytic and theoretical review of quintessential self-regulatory failure. Psychological Bulletin, 133 (1), 65-94. doi: https://doi. org $/ 10.1037 / 0033-2909.133 .1 .65$

Stolarski, M., Wiberg, B. y Osin, E. (2015). Assessing temporal harmony: the issue of a balanced time perspective. En M. Stolarski, N. Fieulaine y W. van Beek (Eds.), Time Perspective Theory; Review, Research and Application: Essays in Honor of Philip G. Zimbardo (pp. 57-71). Berlín: Springer.

Valenzuela, R., Codina, N. y Pestana, J. V. (2020). Relations between university students' physical activity, academic self-regulation and procrastination: Considering motives and gender-differences in the promotion of healthy habits. En L. Gómez Chova, A. López Martínez e I. Candel Torres (Eds.), INTED2020 Proceedings. 14th International 
Technology, Education and Development Conference March 2nd-4th, 2020 - Valencia, España (pp. 7000-7005). Valencia: IATED Academy.

Valenzuela, R., Codina, N., Castillo, I. y Pestana, J. V. (2020). Young University Students' Academic Self-Regulation Profiles and Their Associated Procrastination: Autonomous Functioning Requires Self-Regulated Operations. Frontiers in Psychology, 11, 354. doi: https:// doi.org/10.3389/fpsyg.2020.00354

Van Eerde, W. (2003). A meta-analytically derived nomological network of procrastination. Personality and Individual Differences, 35 (6), 1401-1418. doi: https://doi. org/10.1016/S0191-8869(02)00358-6

Webster, J. D. (2011). A new measure of time perspective: Initial psychometric findings for the Balanced Time Perspective Scale (BTPS). Canadian Journal of Behavioural Science / Revue canadienne des sciences du comportement, 43 (2), 111-118. doi: https:// doi.org/10.1037/a0022801

Wiberg, M., Sircova, A., Wiberg, B. y Carelli, M. G. (2012). Operationalizing balanced time perspective in a Swedish sample. The International Journal of Educational and Psychological Assessment, 12 (1), 95-107.

World Medical Association (2013). World medical association Declaration of Helsinki: ethical principles for medical research involving human subjects. Journal of the American Medical Association, 310 (20), 2191-2194. doi: https://doi.org/10.1001/jama.2013.281053

Yang, X., Xu, X. y Zhu, L. (2015). Media multitasking and psychological wellbeing in Chinese adolescents: Time management as a moderator. Computers in Human Behavior, 53, 216-222. doi: https://doi.org/10.1016/j. chb.2015.06.034

Yuan, B. y Jiang, Z. (2017). Disruption Management for the Real-Time Home Caregiver Scheduling and Routing Problem. Sustainability, 9 (12), 2178 doi: https://doi. org/10.3390/su9122178

Zaleski Z. (1994). Psychology of future orientation. Lublin: Towarzystwo Naukowe KUL.

Zimbardo, P. G. y Boyd, J. (2009, trabajo original publicado en 2008). La paradoja del tiempo: la nueva psicología del tiempo. Barcelona: Paidós.

Zimbardo, P. G. y Boyd, J. N. (1999). Putting time in perspective: A valid, reliable individualdifferences metric. Journal of Personality and Social Psychology, 77 (6), 1271-1288. doi: https://doi.org/10.1037/0022-3514.77.6.1271

Zimbardo, P. G., Keough, K. A. y Boyd, J. N. (1997). Present time perspective as a predictor of risky driving. Personality and Individual Differences, 23 (6), 1007-1023. doi: https://doi.org/10.1016/S0191-8869(97)00113-X

\section{Biografía de los autores}

Nuria Codina Mata es Profesora del Departamento de Psicología Social y Psicología Cuantitativa de la Universidad de Barcelona y miembro del grupo de investigación consolidado PsicoSAO (psicología social, ambiental y organizacional) (2017 SGR 564). Sus líneas de investigación psicosocial se centran en: el tiempo y las actividades de ocio, las experiencias de ocio, el disfrute y la autonomía en la práctica de actividades estructuradas y no estructuradas, la procrastinación y las orientaciones temporales.

\section{iD}

https://orcid.org/0000-0003-0280-3651

\section{Rafael Valenzuela García es Doctor} en Gestión de la Cultura y el Patrimonio por la Universidad de Barcelona. Profesor del Departamento de Psicología Social y Psicología Cuantitativa de la Universidad de Barcelona y miembro del grupo de investigación consolidado PsicoSAO (2017 SGR 564). Investiga desde una perspectiva psico-pedagógico-social la formación de la motivación de calidad en el aprendizaje formal y en el ocio, especialmente en la práctica musical. 
José Vicente Pestana Montesinos en la imbricación de las actividades de ocio es Doctor en Psicología por la Universidad en la cotidianidad de los individuos - con de Barcelona. Profesor del Departamento énfasis en el desarrollo de la persona (hade Psicología Social y Psicología Cuanti- biendo incorporado recientemente la perstativa de la Universidad de Barcelona y pectiva analítica junguiana)—. Especialimiembro del grupo de investigación consolidado PsicoSAO (2017 SGR 564). Su trayectoria científica se ha fundamentado zado en el estudio del teatro. 